\title{
A NEW FORM OF VIBRATION GALVANOMETER
}

\author{
By P. G. Agnew
}

CONTENTS

Page

Abstract......................................... 37

I. Introduction $\ldots \ldots \ldots \ldots \ldots \ldots \ldots \ldots \ldots \ldots \ldots \ldots \ldots \ldots \ldots \ldots \ldots \ldots \ldots \ldots,{ }_{37}$

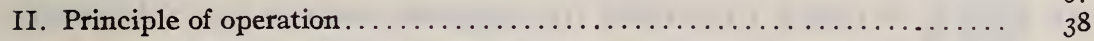

III. Arrangement for greatest sensitivity $\ldots \ldots \ldots \ldots \ldots \ldots \ldots \ldots \ldots \ldots \ldots, 40$

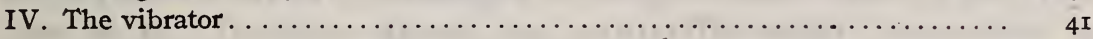

V. Performance....................................... 43

ABSTRACT

Vibration galvanometers are very useful in null measurements, but have not been much used in industrial laboratories on account of their being sensitive to external vibrations and requiring delicate adjustments. The present instrument, which has a sensitivity higher than other forms of the moving-iron type, but less than that of the most sensitive forms of the moving-coil type, has the advantages of sturdiness, quick responsiveness, and freedom from the effects of external vibration. It consists essentially of a fine steel wire mounted on one pole of a permanent magnet and so arranged that the free end of the wire may vibrate between the poles of an electromagnet through which the current to be detected passes.

\section{INTRODUCTION}

The vibration galvanometer is a very useful instrument in alternating-current measurements where null methods can be used, as, for example, in an almost endless variety of bridge measurements, in various applications of the alternating-current potentiometer, and in testing instrument transformers. As in the case of direct-current galvanometers, there are two general types of vibration galvanometers-the moving-coil type and the movingiron type.. In each type the moving element is mechanically tuned so that its natural period is the same as that of the alternating electromagnetic forces produced by the current to be detected, thus using the principle of resonance to produce a relatively large motion for a very small current. ${ }^{1}$

The reading is usually made by means of a telescope or projection upon a screen by observing the image of an electric lamp

\footnotetext{
1 For a general discussion of vibration galvanometers see Laws, Electrical Measurements, I9I 7, p. 434; F. Wenner, Trans. Am. Inst. Elec. Eng., 31, p. I243; F. Wenner, B. S. Bulletin, 6, p. 347, I9ro; A. Campbell, Proc. Phys. Soc., Lrondon, 26, p. 120, 1914.
} 
filament reflected in a very small mirror attached to the moving system. When a current passes through the instrument, the vibration of the moving element causes the image of the filament to appear to broaden into a band.

It is evident that a vibration galvanometer of either type is. simply a specialized form of synchronous motor, the whole mechanical output of which is used in overcoming air friction and elastic hysteresis.

The vibration galvanometer has been used but little in industrial laboratories, its principal use being in physical laboratories, and mainly in precision work. The chief reasons for this limited use are that it is easily disturbed by external mechanical vibrations, and that delicate adjustments are necessary. In the present form of instrument these difficulties are greatly reduced. It is not, however, as sensitive as some forms of the moving-coil type.

\section{PRINCIPLE OF OPERATION}

The present instrument is of the moving-iron type. It consists essentially of a fine steel wire mounted on one pole of a permanent

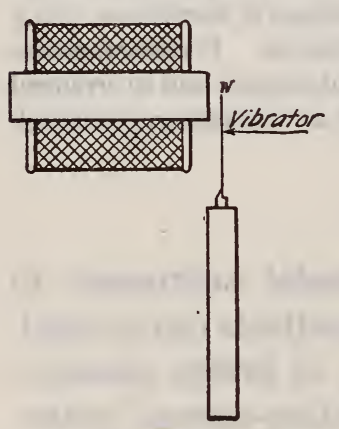

FIG. I. -Illustrating the simple principle magnet and so arranged that the free end of the wire may vibrate between the poles of an electromagnet through which the current to be detected passes. If an unmagnetized steel wire, $W$, is held near the pole of an electromagnet, as in Fig. I, the end of the wire will be pulled toward the pole of the electromagnet during each half wave of the current flowing in the winding of the electromagnet; that is, the wire will vibrate with twice the frequency of the current. If the wire be magnetized by mounting it on the pole of a permanent magnet, the free end of the wire will be alternately attracted and repelled by the alternating flux of the electromagnet; that is, the wire will vibrate with the same frequency as that of the current. But, what is of more importance, the alternating mechanical pull will be very much greater than with an unpolarized wire, because the total flux from the wire is much greater.

The permanent magnet plays the same rôle in increasing the motion of the wire that the permanent magnet in a telephone receiver does in increasing the motion of the diaphragm. The total pull varies as $B^{2}$, and the change in the pull varies as the 
change in $B^{2}$ resulting from the alternating current. Hence if a given small alternating current produces a change in flux, $\Delta B$, the alternating mechanical pull is proportional to $B \Delta B$, instead of to $\overrightarrow{\Delta B}^{2}$, as it would be if the wire were not polarized by the permanent magnet.

Fig. 2 shows one of the first arrangements experimented with. A small electromagnet is mounted on the inside pole face of the permanent magnet, and the finewire vibrator on the opposite pole face. The operation is readily seen. During one half cycle the flux from the pole tip $A$ is slightly strengthened and that through $B$ is weakened. Hence the vibrator moves toward $A$. During the

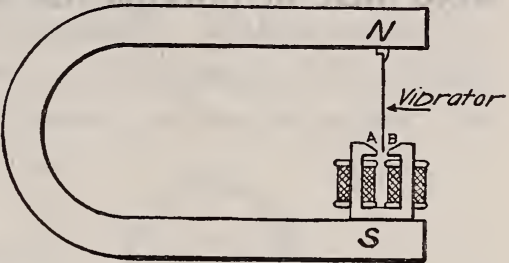

FIG. 2.-An early form of the instrument next half cycle the conditions are reversed and the vibrator moves toward $B$, and so on.

Considering the arrangement as a motor, it is easy to see how the back emf is generated. If the vibrator is moved back and forth by mechanical means, magnetic lines of force from the end of the vibrator move back and forth between $A$ and $B$, alternately increasing and decreasing the flux in each, and thus generating an alternating emf in the winding.

The vibrator, which is, of course, mechanically tuned so that its period is the same as that of the current to be detected, is

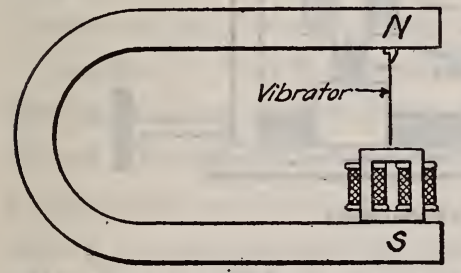

Fig. 3.-Limiting case of no gap in the magnatic circuit of the electromagnet observed by a microscope mounted perpendicularly to the plane of the paper and focused on the end of the vibrator. In this way a much smaller motion can be detected than is possible with the unaided eye.

During a set of experiments undertaken in order to determine optima of conditions, such as size and shape of electromagnet, shape of pole tips, length of gap, and distance between end of vibrator and pole tips, an interesting observation was made. As a limiting case, the air gap of the electromagnet was reduced to zero by using a closed core, as shown in Fig. 3. Even under this condition the device was nearly a tenth as sensitive as with the best arrangement that could be obtained with the same core after it had been sawed so as to form an adjustable gap. 


\section{ARRANGEMENT FOR GREATEST SENSITIVITY}

Of the many arrangements of parts that have been tried, that which gives the greatest sensitiveness and general convenience of working is shown in Fig. 4.

The electromagnet is placed outside the gap of the permanent magnet, but in a position in which an appreciable amount of flux from the latter passes through the cores of the former. It is to be noted that in principle the arrangement is identical with the

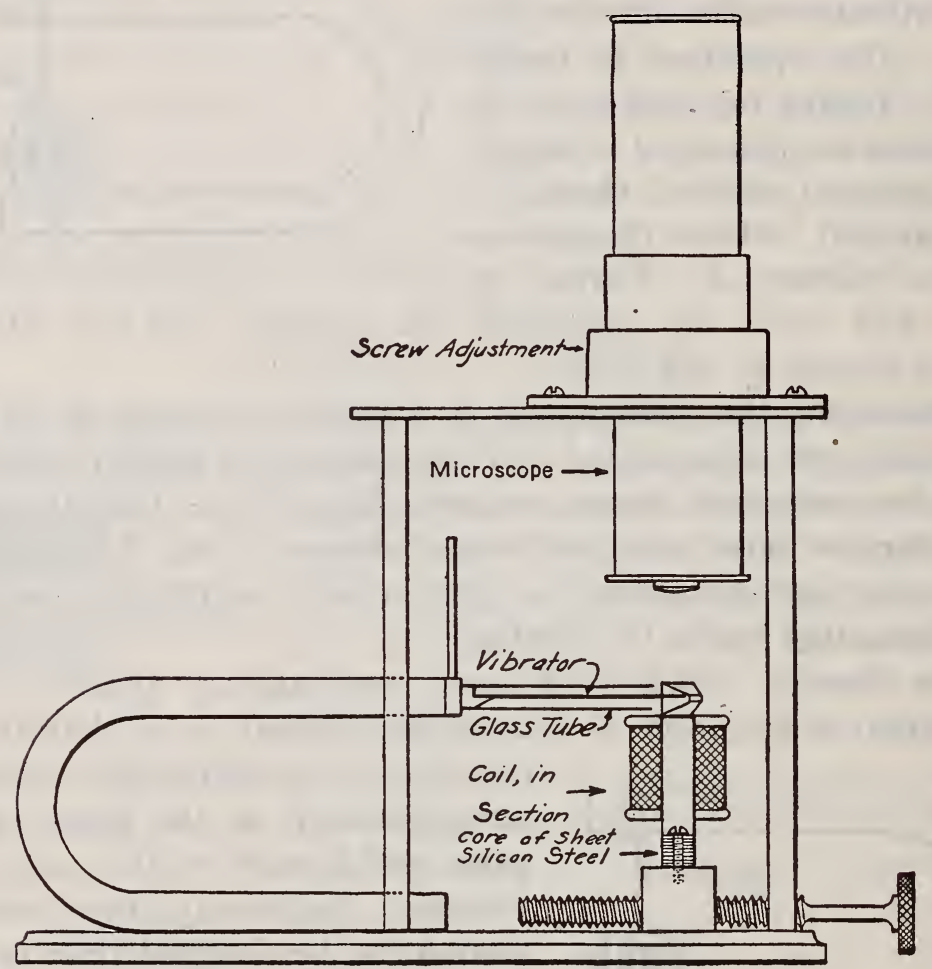

FIG. 4.-The present form of instrument

earlier one described above, the most important difference in the details of the arrangement being the weaker field to which the core is subjected. The two principal advantages of putting the electromagnet outside are: First, other things being equal, the instrument will be most sensitive when the core is working at the point of maximum differential permeability, and the point of maximum differential permeability occurs at very low values of $B$ (ideally, at $B=0$ ); second, with low-frequency vibrators, which must be of very small diameter, the effect of the strong magnetic field upon the frequency of the vibrator introduces practical difficulties in 
tuning, since the magnetic forces combine with the elastic force to change the total restoring force, thus changing the frequency. The relative positions of the permanent magnet and the electromagnet were varied systematically, but, as would be expected, the one shown gave the best results.

A high differential permeability is necessary, as just mentioned, for sensitiveness. This means a high initial permeability in the core. Decidedly the best results have been obtained with cores made of good sheet silicon transformer steel. Results seemed to indicate that the sensitiveness varies as the square of the initial permeability instead of as the first power, as was expected, but sufficiently accurate data were not obtained to make this point certain.

If it is attempted to increase the sensitiveness by bringing the end of the vibrator close to the pole tips, a condition of instability is reached, the vibrator being pulled over to one or the other of the pole tips. Other practical difficulties arise, such as changes in the tuning of the vibrator. In general, the shorter the gap, the closer can the end of the vibrator be placed to the pole tips without such difficulties

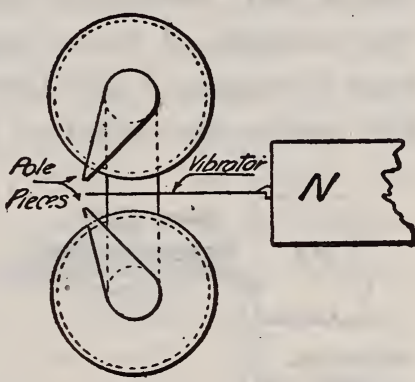

FIG. 5.- Showing slanting of pole tips to increase sensitiveness by increasing field on side on which vibrator is located

being encountered. Considerable changes may be made in length of gap, shape of pole tips, etc., without greatly affecting the maximum working sensitiveness attainable by the various adjustments.

Generally satisfactory results are obtained with the pole tips brought down in the form of truncated pyramids, the faces being about 2 by $0.5 \mathrm{~mm}$, the short edges being parallel with the vibrator, a gap allowed of about $1.5 \mathrm{~mm}$, and the end of the vibrator being about $1.5 \mathrm{~mm}$ from the pole tips. An improvement by a factor of 1.5 to 2 may be obtained by setting the pole tips at an angle, as the field is intensified by such an arrangement. (See Fig. 5.)

\section{THE VIBRATOR}

The vibrator is mounted on a small base of soft steel, as shown in Fig. 6 , and is held in place magnetically by merely placing the base on the face of the magnet. For convenience in changing vibrators for different frequencies, an aluminum wire is inserted in the base for a handle. For a 6o-cycle vibrator a $0.1 \mathrm{~mm}$ wire is convenient, and with this diameter a length of about $33 \mathrm{~mm}$ is 
required. A $0.04 \mathrm{~mm}$ wire of the same length has a frequency of about 25 cycles. For soft-steel wires the following formula is sufficiently accurate for design purposes:

$$
\text { Frequency }=65000 \frac{\text { diameter }}{{\text { (length })^{2}}^{2}}
$$

where the dimensions are in centimeters. ${ }^{2}$

For piano wire the constant is higher, about 70000 .

At first it was hoped that such a vibrator might be permanent enough in its frequency to serve the purpose of a frequency meter as well as that of a galvanometer, but this is not feasible because the actual resonance frequency depends to a small extent upon the position of the vibrator in the magnetic field. Advantage may be taken of this fact, however, to provide a fine adjustment for tuning the vibrator to the exact frequency desired. If an

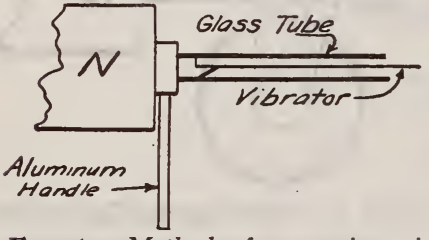

FIG. 6.- Method of mounting vibrator on pole face of magnet auxiliary small magnet, or, better, a pair of such magnets, is placed near the end of the vibrator, the frequency may be changed several per cent by changing the position of the auxiliary magnet. While it does not readily provide as large a range of adjustment, a more convenient method is to move an iron rod toward or away from one pole of the permanent magnet by a screw motion, as shown in Fig. 4.

If the vibrator is polished, and illuminated by a horizontal beam of light, a sharp line of light may be obtained in the microscope, very similar in appearance to the image of an incandescent filament of moderate brilliancy when viewed in a telescope. Satisfactory readings may also be made by viewing the wire in the ordinary way. In comparing sensitiveness it is convenient to use a vibration just sufficient to make the vibrator appear of double diameter.

It is well to keep the vibrator covered with a very thin film of - oil to prevent corrosion. The effect of such a film on the frequency is too small to be at all inconvenient. For many purposes it is convenient to shield the vibrator with a glass tube as in Fig. 6 .

\footnotetext{
2 This formula is in the form given by Rayleigh (Theory of sound, 1, art. $x 7 \mathrm{x}$ ) for the design of the rectangular prong of a tuning fork,

$N=84590 t / l^{2}$

where $N$ is the frequency, $t$ the thickness, and $l$ the length.
} 


\section{PERFORMANCE}

With a magnifying power of 50 to 100 , which has been found satisfactory under working conditions, a motion of the vibrator of 5 microns is easily visible. With a I-ohm winding an emf of 3 microvolts can be detected. The construction is such that high-resistance coils may easily be wound for high current sensitivity. With a $270-0 h m$ winding the sensitivity is such that a current of 0.05 microampere can readily be detected.

The chief advantages of the instrument are its sturdiness, the ease of adjustment, its quick responsiveness, and its freedom from the effects of external vibration. In the last characteristic the instrument is an order of magnitude better than any other form of vibration galvanometer with which the author is familiar. Both the freedom from external vibration and the quick responsiveness are due to the relatively large damping by air friction, and the extremely small mass of the moving element. Although the vibration galvanometer depends upon the principle of resonance, an appreciable amount of damping is necessary to give a reasonably quick response to changes in circuit conditions, and the smaller the mass of the moving element the smaller the amount of energy necessary for a given amplitude, and the more quickly will the requisite amount of energy be supplied from the circuit.

The "resonance range" is about I per cent; that is to say, if the frequency of the current is I per cent above or below the frequency of resonance, the amplitude of vibration will be half as great as at resonance.

The efficiency of the present instrument as a motor is very low, the back emf being only a few per cent of the applied emf. If it should be found possible to increase the electrical efficiency of the device, as a motor, to 50 per cent, the sensitivity would be increased in like ratio, and the instrument would be able to do the work of the most sensitive form of the moving-coil type. Wenner has shown that it is possible to develop an electrical efficiency of over 97 per cent in a vibration galvanometer of the moving-coil type. ${ }^{3}$

The instrument, in various stages of development, has been used in routine testing at the Bureau of Standards for more than three years. While many improvements have been made in detail, a much-to-be-desired radical increase in electrical efficiency has not been accomplished. One possibility is the use of a hardened-steel vibrator, permanently magnetized, but a preliminary

${ }^{3}$ Wenner, B. S. Bulletin, 6, p. 364; rgro. 
substitute experiment, in which a soft-iron vibrator was surrounded by a magnetizing coil, did not show encouraging results. No attempt has been made to put the vibrator in a vacuum so as to reduce the air friction. It would be necessary to use a fairly high vacuum as the resistance of the air is nearly independent of the pressure down to a pressure of about I mm of mercury. The sharpness of tuning necessary would increase as the sensitiveness increased, as would also the response to external mechanical disturbances, especially disturbances synchronous with the current. A very promising line of attack which has not been tried is the use of a vibrator made of vacuum-process iron, to secure, if possible, a much higher flux in the vibrator.

Mr. J. B. Dempsey carried on the larger part of the experimental work and contributed many valuable suggestions.

WASHINGTON, November 24, I9I9. 